\title{
Flight Management System Pathfinding Algorithm for Automatic Vertical Trajectory Generation
}

\author{
Ramon Andreu Altava*, Jean Claude Mere ${ }^{\dagger}$, Daniel Delahaye ${ }^{\ddagger}$ and Thierry Miquel ${ }^{\S}$ \\ ${ }^{*}$ Airbus Operations SAS, Toulouse, France \\ ${ }^{*} \ddagger \S$ Ecole Nationale de l'Aviation Civile, University of Toulouse, France \\ Email: *ramon.andreu-altava@airbus.com, †jean-claude.mere@airbus.com, ${ }^{\ddagger}$ delahaye@ recherche.enac.fr, ${ }^{\S}$ thierry.miquel@enac.fr
}

\begin{abstract}
Air Traffic has evolved over the years with current commercial fleet expected to double in the near future, increasing airspace congestion. Jet fuel prices volatility, market competition and drones integrating the same airspace require a modernization of the Air Transportation System. In this context, Airbus proposes modern avionics and cockpit designs to fulfil these requirements. Flight Management System (FMS), in-service since the early $1980 \mathrm{~s}$, is one of these candidate avionic systems to be revisited. During high workload flight phases as descent and approach are, best-in-class FMSs compute a fixed vertical reference trajectory based on a lateral flight plan and applicable procedures. Then, flight guidance is responsible for tactical replanning so that deviations are absorbed. FMS reference profile is constructed through a series of hypotheses, which could be enhanced by taking into account other factors such as aircraft optimal energy repartition, ATC restrictions, surrounding traffic, wind errors and mass biases. This paper proposes and compares two methodologies based on a modified version of $A^{*}$ algorithm that solves the Optimal Control Problem in the vertical plane. Fixed and variable speed aircraft trajectories are compared in order to quantify the benefits with respect to current FMS design. The problem is formulated through a relaxed point-mass model with a real performance database for a modern commercial aircraft. It accounts for flight constraints as well as aircraft dynamics for trajectory generation. Fuel consumption is optimized without excessively penalizing flight time. These trajectories are compared with those generated by Airbus FMS simulator. Results show that, for modern arrival procedures such as those defined for Continuous Descent Operations, aircraft energy management (potential and kinetic) is enhanced, producing continuously idle trajectories that consume up to $30 \%$ less fuel than current operations, as obtained for this particular procedure. In that case, flight time would be stretched by few minutes yielding to $15 \%$ extra time, whose trade-off still seems interesting from the airline perspective.
\end{abstract}

Index Terms-Aircraft Trajectory Optimization, Optimal Control, Graph Search, Pathfinding, A*

\section{NOMENCLATURE}

$\alpha$

$\gamma$

$\gamma_{T}$

$\theta$

$A T M$

$A T C$

$B B$

$C D A$

$C I$

CIT
Angle of attack

Airmass flight path angle

Total flight path angle

Pitch angle

Air Traffic Management

Air Traffic Control

Branch and Bound

Continuous Descent Approach

Cost Index

Continuously-Idle Trajectory

$\begin{array}{ll}D & \text { Aerodynamic drag force } \\ D P & \text { Dynamic Programming } \\ \dot{E}_{T} & \text { Total Energy Rate } \\ E_{k} & \text { Specific Kinetic Energy } \\ E_{p} & \text { Specific Potential Energy } \\ E_{T} & \text { Energy Height } \\ E S F & \text { Energy Sharing Factor } \\ F F & \text { Fuel flow } \\ F L & \text { Flight Level } \\ F M S & \text { Flight Management System } \\ F P A & \text { Flight Path Angle } \\ g_{0} & \text { Gravitational acceleration } \\ g(n) & \text { Actual cost from start to node } n \\ h(n) & \text { Heuristic function from } n \text { to target node } \\ h & \text { Altitude } \\ m & \text { Aircraft mass } \\ M & \text { Mach number } \\ N L P & \text { Non Linear Programming } \\ O C P & \text { Optimal Control Problem } \\ s & \text { Distance } \\ T h r & \text { Thrust } \\ T S P & \text { Thrust Setting Parameter } \\ V C A S & \text { Calibrated Airspeed } \\ V & \text { True Airspeed } \\ V_{w} & \text { Wind speed }\end{array}$

\section{INTRODUCTION}

Air traffic has evolved over the years and is expected to double by 2030 [1]. According to Airbus global market forecasts [2], 33.000 aircraft will enter into service in the next 20 years, doubling current global aircraft fleet. A direct consequence is that airspace volumes will become increasingly congested, specially in continental areas, which increases both pilot and Air Traffic Control (ATC) workload. Jet fuel prices have presented high volatility in the last decades and are expected to raise in the short term. Airlines market competition makes them to continuously optimize their business routes. Other agents such as drones will gradually integrate the airspace. Major projects as Single European Sky Air Traffic Management (ATM) Research in Europe [3] and Next GENeration (NextGEN) [4] in the United States foster research activities that provide operational solutions to modernize current ATM system. In this context, Airbus proposes new avionics and 
cockpit designs to fulfil these requirements. Flight Management System (FMS), in-service since the early 1980s [5], is one of these candidate avionic systems to be revisited in the near term.

Best-in-class FMSs embarked in Airbus aircraft have been continuously improved over the years, adding new functionalities that aim to reduce pilot workload and operating costs [6]. Among the numerous functions the system has, there is navigation, flight planning (both lateral and vertical), performance computation, conveying guidance commands and provision of information to display systems. For the sake of simplicity, the system separates lateral and vertical segments, iterating over time to improve the quality of the generated trajectory. Focusing on descent and approach flight phases [7], which are those of interest for this paper, whenever an arrival procedure is entered into the FMS, a reference trajectory up to the cruise level is computed backwards (i.e. upstream starting from destination). As a consequence, it results in a complete altitude and speed profile constructed based on a series of hypotheses. This trajectory is a combination of segments subjected to altitude and speed constraints defined in the published arrival procedure. In a second stage, the guidance will engage a set of modes and targets depending of aircraft position with respect to the reference profile, except when laterally off-path. FMS estimates aircraft state all along the flight plan. However, hypotheses done by the FMS are simple and evolve in an uncertain environment, so that predictions are used by pilots only as advisory information. For profile construction, FMS considers either idle or geometric path. Per default, idle segments are used except when an altitude constraint restricts the profile construction. In that case, a straight line up to the altitude constraint is constructed. A flight path angle comparison method is adopted in case that airbrakes are needed. In general, geometric segments need extra thrust to keep a constant-speed flight path, deviating from idle setting. FMS computes an optimal descent speed considering a Cost Index (CI), a ratio between time and fuel costs [8]. On one hand, time costs are frequently attributed to maintenance, delays, marginal depreciation, leasing costs and personnel. On the other hand, fuel cost is subjected to market price fluctuations and may vary significantly among geographic sectors.

Regarding to the approach path construction, it is also computed backwards and mainly depends of the type of approach. Per design, flap/slat settings are changed at minimum speed, so that high-lift (and drag) configuration is delayed as much as possible. However, in real operations, aircraft deviate from their intended route and speed as a consequence of ATC instructions, wind errors or separation assurance resulting in an over or under energy aircraft state. Those scenarios require pilot manual intervention, usually through airbrakes, thrust levers and anticipating flap or landing gear extension, in order to recover the right energy state. This is known as energy management in approach and the outcome depends exclusively of the pilot ability.

Modern arrival procedures are based on Continuous Descent
Operations (CDO) paradigm [9], which assumes aircraft deceleration while descending instead of traditional step-down operations. The main advantage of this approach is to achieve an engine thrust-setting close to idle and avoid deceleration segments at low altitudes, which reduces fuel consumption, noise and gas emissions. In order to decelerate whilst descending, an energy repartition factor (between kinetic and potential energy loss) is defined. It is called the Energy Sharing Factor (ESF) and, for current trajectory design, its value is fixed and exclusively considered for decelerating segments. It seems reasonable to say that, depending of the flying procedure, a variable ESF may produce a better outcome. The same applies for the approach path. Potentially, it might improve current FMS descent path construction as it reduces the probability of a vertical discontinuity, which is not a very recurrent issue, and smooths Continuous Descent Approach (CDA) profile construction.

Aircraft trajectory optimization is frequently modelled as an Optimal Control Problem (OCP). Numerical approaches were popularized in lieu of analytical solutions based on calculus of variations theory. In general, OCPs can be classified in two categories: direct and indirect. Studies such as [10] [11] [12] reunite and compare those methods, analysing the advantages and drawbacks of each approach. In summary, indirect methods rely on first-order necessary conditions and Pontryagin's Maximum Principle [13], a prohibitive approach for complex and highly-constrained non-linear problems. Direct methods transcript the OCP into a Non-Linear Programming (NLP), which avoids explicit derivation of the necessary conditions. To solve it, pseudo-spectral collocation methods have been particularly popularized [14]. Dynamic Programming (DP), includes those methods that use recursively solutions of simpler sub-problems [15]. Indirect methods for real-time trajectory generation are explored in [16] and compared with Vertical Navigation (VNAV) FMS construction. Time and Energy Management Operations (TEMO) [17] proposes a pseudospectral algorithm that constructs energy-neutral trajectories (i.e. neither additional thrust nor speed brakes extension), based on Continuous Descent Operation strategy, with fixed arrival time. The function absorbs tactical deviations or recomputes the profile (strategic) but it only covers nominal scenarios and the flight is divided in multi-segments. Regarding the family of DP and Branch\&Bound (BB) algorithms, a set of graph search techniques for general aviation trajectory generation is presented in [18]. They propose techniques for speeding-up those algorithms including heuristics, obtaining disparate results. In particular, $\mathrm{A}^{*}$ heuristic algorithm has been successfully applied in [19] and [20]; however, those works define a priori the state space and adopt simple heuristics that penalize the flexibility and optimality of the solution. Beam search algorithm combined with a search space reduction method is proposed in [21] for end-to-end route optimization, based on free-flight concept. Significant gains are obtained when compared with a FMS, nonetheless, ATC constraints shall be added to improve the representativeness of those results. Soft Dynamic Programming algorithm was proposed 
in [22] for optimizing altitude and speed profiles during cruise for initial-4D (i4D) operations. State space pruning and neural networks implementation permitted to reduce algorithm timecomplexity to acceptable levels.

To conclude, the main pain-point of the works found in the literature is the lack of generality of those algorithms. Most of the approaches, either do not consider actual procedure constraints or they divide the problem in multi-stages that correspond to 'typical' descent profiles. As a consequence, results are rarely extrapolated to similar problems. Other researches instead, do not prioritize real-time basis for ad-hoc optimal trajectory computation. The main contribution of this paper is that it aims to go further than state-of-the-art CDO and it searches an alternative to constant-speed descents that proves the benefit of variable-speed paths. Fully-idle trajectories are possible if energy repartition strategy is better adapted to the procedure itself. Nonetheless, this study does not intend to prove that this is true for all kind of arrival procedures but that it is for most of them and that there is room for improvement in current flight trajectory construction. Furthermore, this paper is innovative from an algorithmic standpoint, since typical $\mathrm{A}^{*}$ based algorithms applied to trajectory optimization problems define a grid containing all possible states with a fixed branching factor, which differs from this paper where states are developed though a discretized control that represents an action. As a consequence state values are not imposed a priori but gradually explored.

The paper is divided as follows; Section II formulates mathematically the problem and the set of associated constraints. Justification for the choice of methodology and a review of $A^{*}$ formal basis is exposed in Section III. Use case definition and analysis of the results for constant and variablespeed descent operations is covered in Section IV. Eventually, Section $\mathrm{V}$ presents final conclusions and outlines future work and perspectives.

\section{Mathematical Model}

\section{A. Problem formulation}

FMS uses a series of lookup tables embedded in a Performance database (PDB) that contain engine, aerodynamic and performance models. A customized version of this PDB is used for that of a Single Aisle aircraft. These data are frequently a combination of analytic and experimental results. The algorithm uses a set of calling routines to extract required data by means of linear, bilinear or trilinear interpolation methods, depending of grid dimension. In steady-flight, equilibrium is achieved through equation (1). Required thrust is then used for iterating an engine-specific scaling parameter, also known as Thrust Setting Parameter (TSP) and then, compute fuel flow through equation (3):

$$
\begin{gathered}
T h r=D+m \sin \gamma\left(g_{0}+V \frac{d V}{d h}\right) \\
\operatorname{Thr}(M, T S P) \\
F F(h, M, T S P)
\end{gathered}
$$

The objective function to be minimized along the whole trajectory is the flight cost, a trade-off between fuel consumption and flight time, which has the form of:

$$
J=\arg \min \int_{s_{0}}^{s_{f}}\left(F F+\frac{C I}{60}\right) \frac{1}{V \cos \gamma+V_{w}} d s
$$

where $C I$ is expressed in $\frac{\frac{\$}{m i n}}{\frac{\$}{K g}}=\frac{K g}{\min }$ and $F F$ in $\frac{K g}{s}$.

This paper presents an approach that solves aircraft trajectory optimization problem through a combination of Optimal Control Problem (OCP) and pathfinding techniques, whose goal is to find the best set of actions that generate an optimal trajectory. On this basis, this study compares two kind of trajectories; on one hand, those generated with fixed speed and, on the other hand, trajectories constructed with variable speed at idle-thrust.

\section{B. Aircraft equations of motion}

State variables are defined through relaxed point-mass aircraft equations of motion in the vertical plane, which ensures an appropriate compromise between representativeness and complexity [23]. Time-dependent equations can be formulated as distance-dependent by multiplying per $\frac{d t}{d s}=\frac{1}{V \cos \gamma+V_{w}}$. This hypothesis is particularly useful as most constraints are typically distance-dependent, what eases their manipulation. It leads to the following formulation of aircraft equations of motion:

$$
\begin{gathered}
s^{\prime}=\frac{d s}{d s}=1 \\
h^{\prime}=\frac{d h}{d s}=\frac{V \sin \gamma}{V \cos \gamma+V_{w}} \\
V^{\prime}=\frac{d V}{d s}=\frac{T h r-D-m g_{0} \sin \gamma}{m\left(V \cos \gamma+V_{w}\right)} \\
m^{\prime}=\frac{d m}{d s}=\frac{-F F}{V \cos \gamma+V_{w}}
\end{gathered}
$$

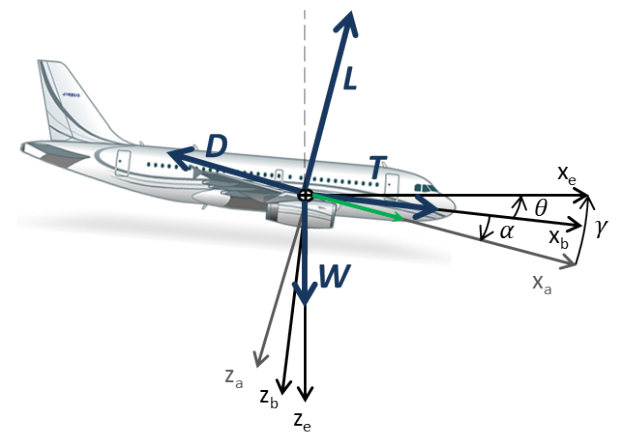

Fig. 1. External Forces on Aircraft During Descent Phase

It has to be noted that for simplification, wind gradients have been neglected and thrust is considered co-linear with the velocity vector, so that $T h r \cos \alpha \approx T h r$. 
Energy Sharing Factor, already defined in previous section, represents how the total energy loss is repartitioned between potential and kinetic energy. This paper assumes that in descent the total energy rate is negative (9), since drag is greater than thrust, which is imposed to idle:

$$
\dot{E}_{T}=\frac{\left(T h r_{i d l e}-D\right) V}{m g_{0}}<0
$$

Definitely, $E S F$ can be formulated as:

$$
E S F=\frac{\dot{E}_{k}}{\dot{E}_{k}+\dot{E}_{p}}
$$

Taking into account that energy height rate, which is independent of aircraft mass, is defined as the sum of kinetic and potential energy rates:

$$
\dot{E}_{T}=\dot{E}_{k}+\dot{E}_{p}=V \sin \gamma+\frac{V \dot{V}}{g_{0}}
$$

Combining (10) and (11) the following expression is deducted:

$$
\sin \gamma=\frac{(1-E S F)}{E S F} \frac{\dot{V}}{g_{0}}
$$

In order to simplify the equations, the total flight path $\left(\gamma_{T}\right)$ is defined as the sum of aerodynamic flight path angle and the respective acceleration / deceleration:

$$
\sin \gamma_{T}=\sin \gamma+\frac{\dot{V}}{g_{0}}=\frac{\sin \gamma}{1-E S F}=\frac{(T h r-D)}{m g_{0}}
$$

Eventually, considering that $\sin \gamma \approx \gamma$ and $\cos \gamma \approx 1$, aircraft equations of motion (6) and (7) result in:

$$
\begin{gathered}
h^{\prime}=\frac{V(1-E S F) \gamma_{T}}{V+V_{w}} \\
V^{\prime}=\frac{g_{0} E S F \gamma_{T}}{V+V_{w}}
\end{gathered}
$$

\section{Flight constraints}

Constraints on state variables are dictated by arrival procedures design. They are contained in the navigation database (NDB) under the form of altitude and speed constraints, coded in ARINC 424 standard by database providers. They impose that aircraft altitude shall remain below, above, in-between or at a certain level when it applies. A speed constraint dictates that the aircraft shall be lower than a certain speed. Furthermore, ATC regulation generally imposes a maximum speed of $250 \mathrm{kts}$ Calibrated Airspeed (CAS) for all aircraft below FL100.

Control value $\gamma$ is bounded to a maximum and minimum value; $\gamma_{\max }$ is upper-limited to zero so that no climb segment is constructed, since it would not be operationally acceptable. Nevertheless flight levels may be constructed if optimal. As for the lower bound, aircraft descent capability is limited to a $\gamma_{\min }$. This value is fixed or variable, with lower values accepted for accelerated descents:

$$
\gamma_{\min } \leq \gamma \leq \gamma_{\max }
$$

In addition, aircraft speed shall remain within the flight envelope for any flap/slat configuration, so that:

$$
V_{C A S_{\min }} \leq V_{C A S} \leq V_{C A S_{\max }}
$$

Passengers comfort is taken into account by limiting longitudinal acceleration / deceleration to a certain g-force.

$$
|\dot{V}| \leq 0.07 g_{0}
$$

Finally, $E S F \in[-1,1]$ as a consequence of equations (16) and (18) for the variable speed sub-problem. For instance, an $E S F=1$ leads to a decelerated level-flight, while an $E S F=$ -1 means that kinetic energy is gained as a consequence of drastic potential loss. $E S F=0$ leads to a loss of altitude at constant true-airspeed.

\section{Methodology}

\section{A. Choice of the methodology}

Pathfinding algorithms have been identified as a promising way to solve real-time trajectory optimization problems when compared with other kind of methods, notably direct and indirect. The main disadvantage of pathfinding algorithms, cited in [15] as 'curse of dimensionality', is related to the number of possible combinations available as the problem grows. However, large set of constraints palliate this issue, since they reduce the search space. In general, BB algorithms handle constraints much easier than indirect and direct metrics. Stochastic algorithms such as Evolutionary, Swarm and Simulated Annealing have been discarded due to certification issues.

Graph search algorithms solve part of those problems since handling constraints is easy and beneficial because it produces large search space reductions. Dijkstra's and Bellman-Ford techniques lead to optimal solutions but they usually explore more nodes than informed algorithms, which use an heuristic function to guide the algorithm towards the target. Basically, A* is a combination of Dijkstra's and greedy best-first search what makes it tremendously interesting for problems where many information is known. $A^{*}$ basis [24] assures optimality whenever the heuristic function is consistent $(f(n)$ monotonically decreasing) and admissible $(h(n)$ underestimates the actual cost). The former is accomplished through the triangle inequality displayed in figure 2 :

$$
h(n, t) \leq g\left(n, n^{\prime}\right)+h\left(n^{\prime}, t\right)
$$

where $n$ is the frontier node, $n^{\prime}$ is a children and $t$ is the target node.

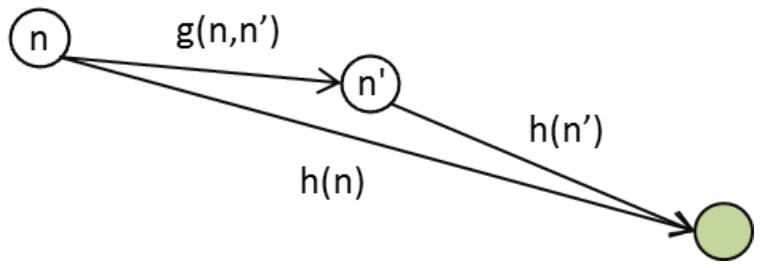

Fig. 2. Triangle Inequality Condition for Consistent Heuristics 
To summarize, the heuristic function is an estimation of the optimal trajectory cost. It has to be underestimated and the more accurate it is, the fewer nodes will be expanded. However, if the actual cost is very precisely-estimated, the solution is somehow known a priori.

A* graph search algorithm manages a list of candidate nodes (Open list) and a second list containing those nodes already explored (Closed list). At each iteration, the node with least cost is developed. Here, cost is defined as the sum of actual and heuristic costs:

$$
f(n)=g(n)+h(n)
$$

where $g(n)$ is the cost from start to node $n$ and $h(n)$ is the estimated cost from node $n$ to the target.

If the algorithm arrives to the same node from another path and this node is in the list Open, it compares the cost, keeps the smaller, updates its parent and puts the node in the Closed list. However, if a node is already in the Closed list, any node coming from any path will be systematically discarded. In this way and considering a consistent heuristic, if the children node is the target, the optimal trajectory is reached and the algorithm stops its search. As each node in the Closed list has registered its parent node, the algorithm can easily track all the nodes pertaining to the optimal trajectory.

\section{B. Algorithm description}

The algorithm is composed of two parts; firstly a backwards integration is performed to define the stopping criterion of the A* part, based on approach chart and a fixed ESF. Then, the $A^{*}$ algorithm starts a forward search that ends whenever the target node is achieved. That said, $A^{*}$ finds an optimal trajectory for the descent path based on a pre-defined approach path. The combination of both forms the full arrival trajectory. The paper focuses mainly on the descent path, which is computed by the $\mathrm{A}^{*}$ algorithm. The problem is represented

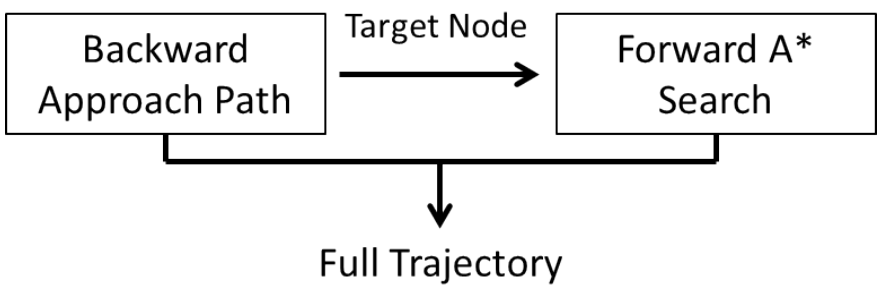

Fig. 3. Algorithm Functional Schema

with a graph that contains a series of nodes and edges $G(n, e)$. A children node is the consequent state produced by an action (control variable). The cost from frontier to children node represents an edge of the graph and is computed according to equation (4). The graph is directed, acyclic and unidirectional. Initially, it is unknown except for the initial and target (end of approach path) points, which define a two-point boundary value problem. The nodes are created according to a certain continuous variable that is discretized; it is the control variable of the problem. An upper and lower limit define a range for the control variable so that bang-bang control is avoided. Nevertheless, constraints on control variable applies as described in section II-C. Children nodes are created as the result of a certain control value. If any of the children nodes falls out of the search space zone, which has been pruned by the set of problem constraints, that node is removed as shown in figure $4 \mathrm{a}$. In addition, as nodes are developed progressively through actions, it is likely that one node fails "too close" to another already in the list. In that case, the node is considered as the adjoin of another, which defines a neighbourhood zone through the tolerance $(\mathcal{E})$ as in figure $4 \mathrm{~b}$. It prevents the algorithm to reconsider nodes that have already been explored. Then, the algorithm removes the node if it is already in the Closed list and updates the cost in case that the node is in the Open list.



(a) Node Out of the Search Space

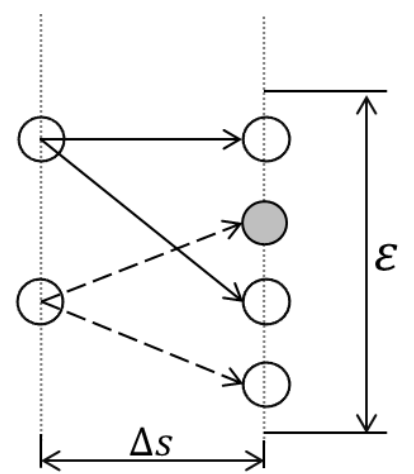

(b) Adjoint Nodes within the Neighbourhood Zone $(\mathcal{E})$
Fig. 4. Frontier Node Expansion Process

Nodes are developed taking into account fixed discretization step, which does not takes into account altitude constraint location. In order to reduce this issue, additional nodes are created at each altitude constraint distance. It means that if an edge traverses the altitude constraint, the intersection is defined as a new node as it is displayed in plain grey in figure 5. It represents real operations, since pilots adapt their flying strategy whenever an altitude constraint is sequenced. The algorithm keeps developing nodes until any node falls within a convergence zone, sufficiently near from the target to be considered as acceptable. This zone is set around the initial approach fix determined by the approach path, which is constructed by means of a backwards Runge-Kutta integration.

\section{RESULTS AND FINDINGS}

\section{A. Use case description}

The test scenario that has been chosen corresponds to that of a CDA Standard Arrival Procedure (STAR) at Blagnac (Toulouse) airport, 14-R runway. It contains three altitude constraints: 'AT OR BELOW' $15.000 \mathrm{ft}$ at NARAK, 'WINDOW' between $11.000-8.000 \mathrm{ft}$ at ABLIS and 'AT OR ABOVE' $3.000 \mathrm{ft}$ at IO14R, which contains at the same time a speed constraint of maximum $215 \mathrm{kts}$. A CDA procedure is 


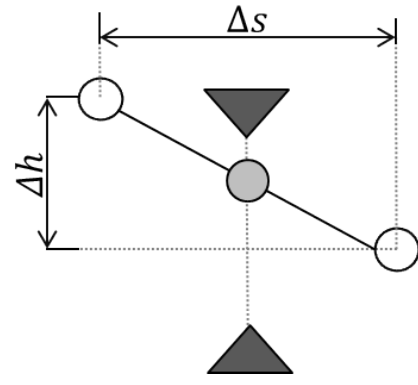

Fig. 5. Created Node at Altitude Constraint Distance

a pertinent selection as it is a modern design that provides a certain degree of flexibility to optimize the profile. On the opposite side, old-fashion procedures imposing level-flight segments restrict the profile optimization.
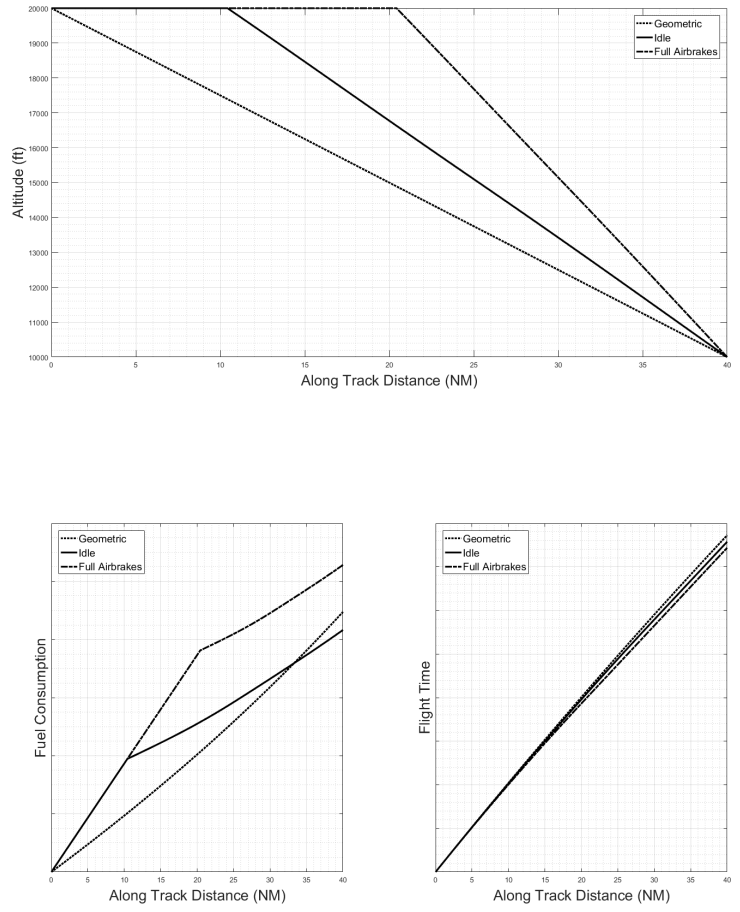

Fig. 6. Flight Performance Trajectory Comparison

In order to define a good heuristics, several type of trajectories have been studied. In a first time, this study is focused on fuel consumption optimization, so that CI defined in (4) is equal to zero. Given two points with known altitudes and distance between them, several trajectories can be constructed; a geometric path linking both altitudes with a straight line, a level-flight plus an idle path and a level-flight plus an idle path with full airbrakes extended. Results showed in figure 6 infer that level-flight plus idle paths are the best in terms of fuel consumption and flight time. The latter is trivial as geometric segments use a thrust lever different than idle, while a level-flight needs extra thrust to keep the speed target but is compensated through the idle segment. Airbrakes-extended trajectory demonstrates that this strategy is only interesting for over-energy scenarios where energy state cannot be recovered without extra drag. However, from a time perspective levelflight plus idle path is as well the most interesting as ground speed is larger at higher altitudes due to the effect of air density in drag. Previous conclusions have also been confirmed in the presence of both tail and head winds and for different waypoint configurations. In conclusion, for constant-speed trajectories, the optimal will be achieved as long as the aircraft is flying at the highest altitude. Consequently, well-defined heuristic functions shall take the conclusions of this study into account.

Section IV-B presents the results that have been obtained for both fixed and variable speed descent and approach operations. All simulations have been performed on a $8 \mathrm{~Gb}$ RAM EliteBook Hewlett Packard computer with Intel Core i5-6300U.

\section{B. Vertical Plane with fixed Speed}

This case considers aircraft descending at optimum speed (here $340 \mathrm{kts}$ ) and at $250 \mathrm{kts}$ from FL100, as per regulation. Consequently deceleration segments are not considered during the whole descent and aircraft passes from optimum speed to $250 \mathrm{kts}$ instantaneously, which is far from being realistic. It reflects a real operation except for the fact that levelflights are allowed if optimality dictates so (nowadays not permitted by construction). Since the optimal trajectory can be deducted a priori through flight performance analysis, a lower bound (i.e. heuristic) can be computed by a backward integration. However, since a full backwards integration is computationally expensive, each node computes the cost from their current position to the fixed bound plus the known cost of the heuristic. Results displayed in figure 8 show that aircraft

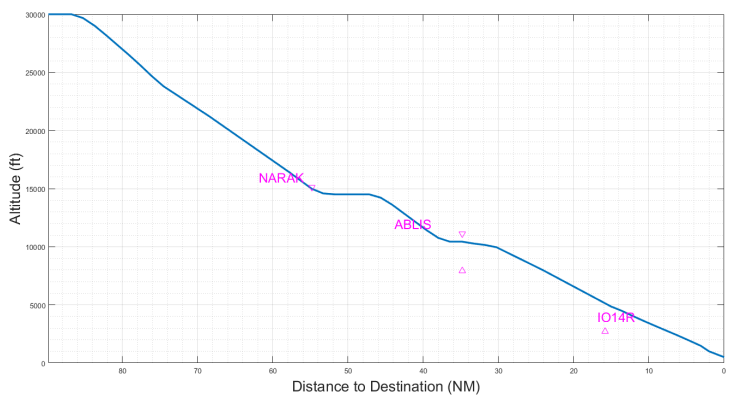

Fig. 7. Optimal Altitude Profile for Fixed-Speed

tend to fly a level-flight then descending idle to honour any altitude constraint. It permits to add thrust during the levelflight but descend at idle-setting. A geometric segment, would have consumed more fuel as thrust is added during the whole path in order to keep the target speed as concluded in previous section. In addition, for the same true airspeed it takes longer as ground speed is larger at higher altitudes.

In this case, the optimization problem has been reduced to find a flyable path with a known lower bound. Gamma value, which is shown in figure 9, is bounded at each iteration to 


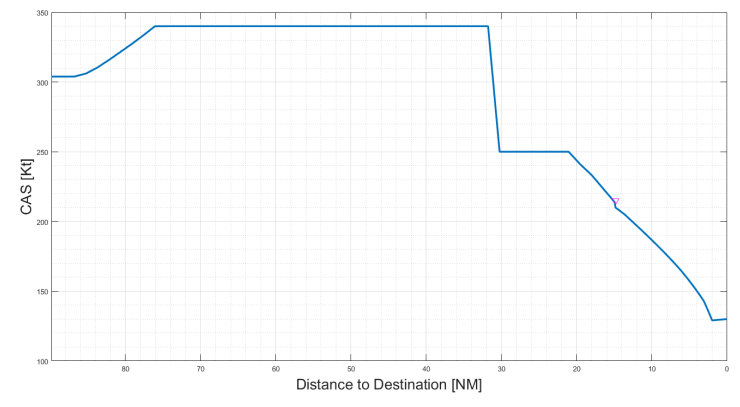

Fig. 8. Optimal Speed Profile for Fixed-Speed

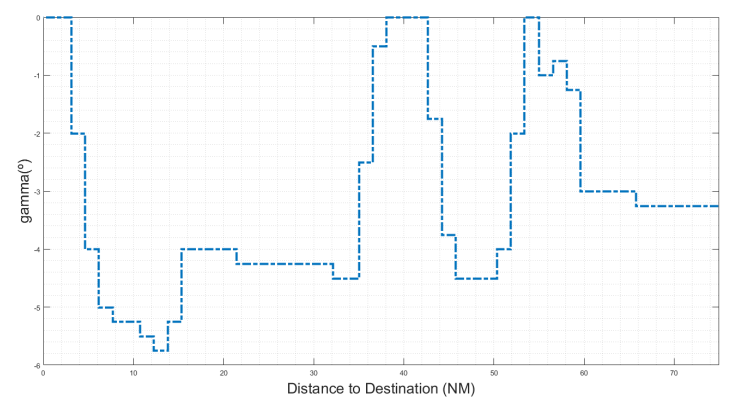

Fig. 9. Commanded Flight Path Angle

avoid bang-bang control and take into account flight controls dynamics, which explains why gamma steps slightly. Fuel consumption evolution shown in figure 10 depicts that levelflight segments require additional thrust to keep the target speed. Flight time is stretched as soon as aircraft decelerates. For fairer comparison, decelerations should be taken into account instead of instantaneous change of speed. Note that for confidentiality reasons no actual values are displayed.

\section{Vertical Plane with Variable Speed}

Unlike the previous case, whose optimal trajectory can be deducted a priori, in this case the result is less obvious to anticipate. For this type of operation, idle-thrust setting is considered during the whole descent and approach. It defines a kind of Continuously-Idle Trajectory (CIT) where optimal energy management is achieved in order to optimize fuel consumption while satisfying all altitude and speed constraints. Aircraft are allowed to reduce and increase their speed whilst it remains within the flight envelope. Accelerations and decelerations limits are established to assure proper passenger comfort.

The full profile is constructed forward and backward as shown in figure 12. First, the approach is constructed by backward integration, considering a -3 FPA at $V_{a p p}$ up to the stabilization altitude (1000 ft) plus an acceleration (upstream) with all aerodynamic changes and a determined ESF of 0.3. The end state is then used as target node for the A* algorithm, which performs a forward search. From figure 12, it can be seen that the aircraft decelerates as it losses altitude slowly,


Fig. 10. Fuel Consumption and Flying Time Evolution for Fixed-Speed

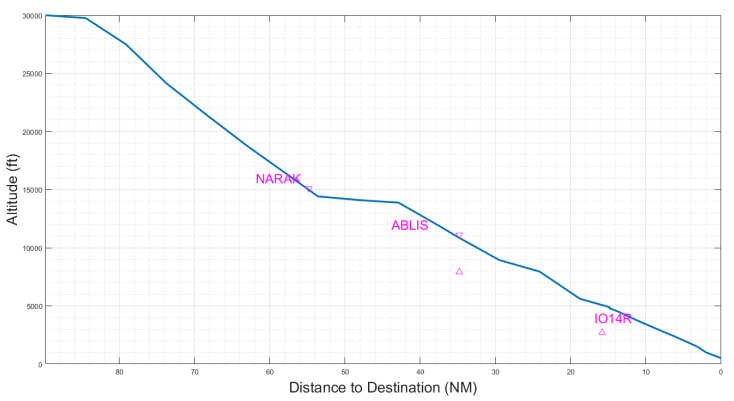

Fig. 11. Optimal Altitude Profile for Variable-Speed

then re-accelerates up to maximum speed. Once NARAK is sequenced, it keeps altitude so that speed is compromised. When FL100 is achieved, what occurs several miles away from ABLIS, speed is limited to 250 kts. From that altitude down to 3700 feet, where approach starts, it performs a continuous deceleration to end at the right conditions.

Energy repartition is displayed in figure 13. In general, negative values are targeted when the aircraft descends, since a steep descent provokes an increase of kinetic energy, while positive values are achieved during decelerated level-flight. Finally the approach is performed at 0.3 ESF, i.e. $30 \%$ of the total energy loss is used for deceleration while $70 \%$ is dedicated to descend. Figure 14 displays a parabolic progression of fuel burnt due to the fact that fuel flow for idle-thrust increases progressively at lower altitudes. Flying the final approach with FPA -3 in landing configuration consumes a relatively high quantity of fuel with respect to the descent part. Flight time slope increases as aircraft decelerates.

\section{Results Comparison}

The previous results are compared with the trajectory constructed by an Airbus FMS simulator as it is displayed in figure 15 , which for comparison purposes is sufficiently representative of a real system. Again, for confidentiality reasons, no actual performance values are displayed. It can be observed that constraints do not restrict the trajectory, so that the path is fully-idle, which is the best case for nowadays design. Since the approach path is not optimized but imposed, focus is placed on the descent path. Fuel consumption and flight time for 


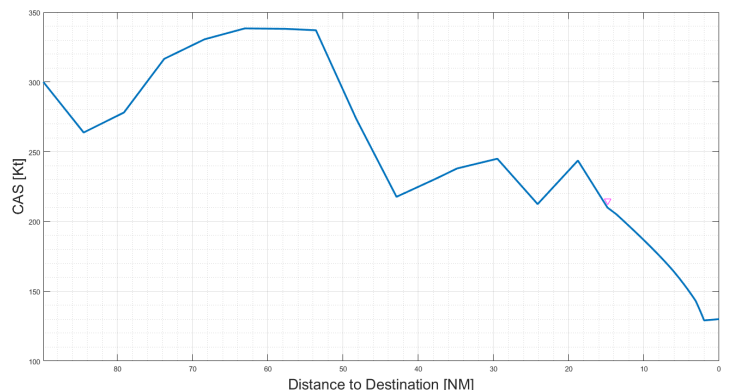

Fig. 12. Optimal Speed Profile for Variable-Speed

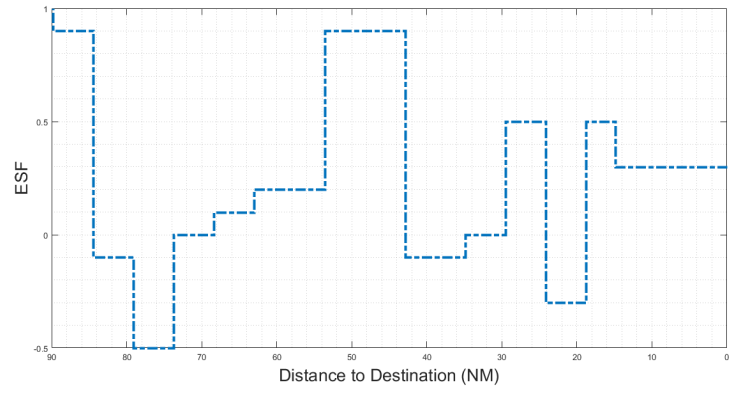

Fig. 13. Commanded Energy Sharing Factor

this calculation is used as reference values for comparison purposes. When compared with the fixed-speed trajectory, a fuel consumption delta is observed, which is due to the fact that the deceleration is performed instantaneously. In fact, two idle paths with same speed and hypothesis should not produce different results. Then, any disparity is due to the modelling and not to the trajectory in itself. Thus, it can be inferred that both trajectories produce the same results. However, when compared with the variable speed method, further analysis is required. Regarding fuel consumption, which is the variable to optimize, variable-speed path reduces fuel burnt as aircraft trades speed by altitude, yielding for this case a 30\% of fuel savings. Nevertheless, flight time is stretched as result of decelerations representing a $15 \%$ extra time. It has to be noted that fuel savings could be even greater for trajectories
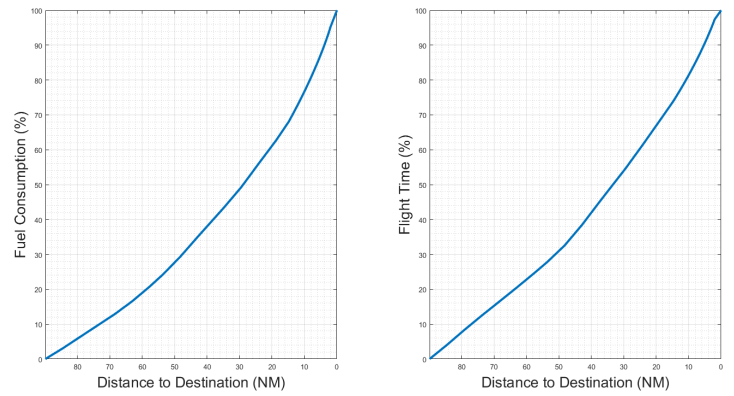

Fig. 14. Fuel Consumption and Flying Time Evolution for Variable-Speed

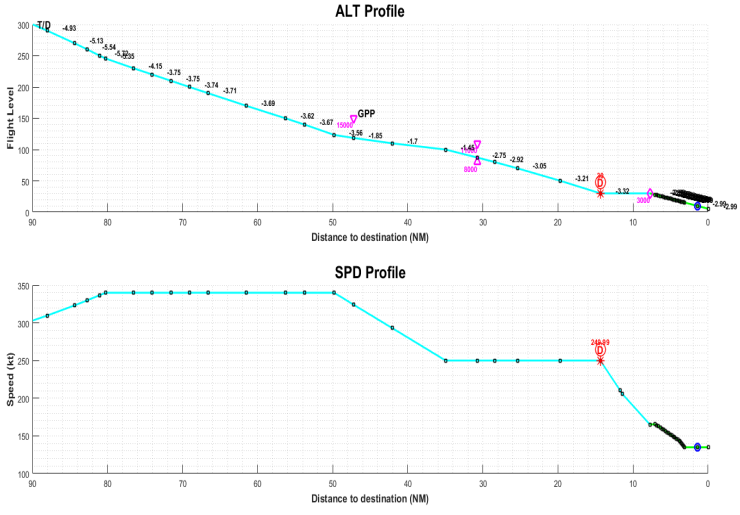

Fig. 15. Flight Trajectory computed by FMS simulator

constructed with several geometric segments.

\section{COnClusions And Future Work}

This paper presents and compares two paradigms in order to optimize aircraft vertical path during descent and approach based on fixed and variable-speed path construction. The algorithm is based on Optimal Control theory that is solved through a modified $\mathrm{A}^{*}$ algorithm, which implements all kind of operational constraints attributed to those flight phases. While fixed-speed paths aim to minimize the use of thrust for an imposed airspeed, variable-speed trajectories intents to apply energy management concepts for an aircraft flying at imposed idle thrust, continuously trading between potential and kinetic energy. All trajectories are compliant with published arrival procedures. A CDO arrival procedure at Blagnac airport has been selected as test scenario. For this case study, results demonstrate that, from an energy management standpoint, variable-speed trajectories reduce fuel consumption by a $30 \%$ while adding only few extra minutes on average to the total flight descent when compared with current design (around $15 \%$ extra time). Idle-thrust has been imposed as it reduces not only fuel consumption but noise and gas emissions. As a consequence, continuously-idle trajectories could be seen as an extension to state-of-the-art CDA function embedded in the FMS. However, flight times are usually stretched so that limits should be imposed to accept the trade-off between time and fuel. The operational impact of this kind of profiles has to be evaluated accurately. These gains prove the potential of the concept in itself, which should be applied to other test procedures for better assessment and validation.

Since the full vertical trajectory is obtained from the combination of a descent and approach path, which could be optimized together, global optimality is not ensured. This pseudo-optimal trajectory could be enhanced if A* algorithm is launched for the whole path. In order to define the top of descent and assure that the aircraft arrives with the proper energy state, $A^{*}$ could be launched backwards from the destination. Furthermore, current version of the algorithm should be improved to produce meaningful results in a reasonable time 
for all kind of procedures. Other improvements that could be explored in the future is to couple the lateral and vertical path, resulting in 3-D or even 4-D trajectories if time is considered. That assumption would provide an extra of flexibility to the algorithm, since some over-energy scenarios could be solved by means of lateral path stretching. Nevertheless, the current algorithm proposes an enhanced alternative to nowadays FMS path construction. Thus, it does not take into account uncertainties coming from ATC instructions, wind errors, gusts, surrounding aircraft and weather avoidance. A future improvement envisages to compute an optimal trajectory when the aircraft is off-path. In that case, which results in over or under energy state, airbrakes, landing gear and high-lift devices extension might be anticipated to recover the proper energy level. A function that generates an ad-hoc trajectory based on these changing conditions would provide a great aid to pilot decision-making or, in full automated mode, optimal energy management in any condition. This complex problem has to be addressed in the near future and may alter the manner optimal trajectories are currently computed in this paper.

\section{REFERENCES}

[1] EUROCONTROL. "Long-Term Forecast: IFR Flight Movements 20102030”. EUROCONTROL, Rep. CND/STATFOR Doc-415, 2010.

[2] AIRBUS, "Global Market Forecast 2018-2037 - Global Networks, Global Citizens" 2018.

[3] SESAR. "European ATM Master Plan". Publications Office of European Union, Edition SESAR JU \& SESAR Work Package C and Partners, Excecutive View, 2015.

[4] NextGen Office. "FAA's NextGen Implementation Plan 2016". Tech. rep-800. FAA, Independence Avenue, Washington, DC, 2016.

[5] S. Liden."The Evolution of Flight Management Systems". Honeywell Air Transport Systems, PO Box.1, Phoenix AZ 85036.0-94 \$4.00IEEE Vol.21, 11-7803-24254, 1994

[6] D.Avery."The Evolution of Flight Management Systems". February, 2011.

[7] AIRBUS "Aircraft Energy Management during Approach" Flight Operations Briefing Notes. October, 2005.

[8] AIRBUS Customer Services. "Getting to Grips with the Cost Index" Flight Operations Support \& Line Assistance. Issue II - May, 1998.

[9] H. Fricke, C. Sei, and R. Herrmann. "Fuel and Energy Benchmark Analysis of Continuous Descent Operations", Air Traffic Control Quarterly, Vol. 23, No. 1 (2015), pp. 83-108.

[10] Betts, J. T. "Survey of Numerical Methods for Trajectory Optimization". Journal of Guidance, Control, and Dynamics, Vol.21, No.2, 193-207, March-April 1998.

[11] D. Delahaye, S. Puechmorel, P. Tsiotras and E. Feron."Mathematical Models for Aircraft Trajectory Design : A Survey". Lecture notes in Electrical Engineering. 290 (Part V), pp 205-247. HAL-00913243. Vol.10, 1007, 431-54475. 2014.

[12] A. V. Rao."A Survey of Numerical Methods for Optimal Control". AAS 09-334.

[13] L. S. Pontryagin and V. G. Boltyanski and R. V. Gamkrelidze and E. F. Mischenko."The Mathematical Theory of Optimal Processes". New York, NY: Interscience Publishers, 1962.

[14] Hargraves, C. R. and Paris, S. W." Direct trajectory optimization using nonlinear programming and collocation" ,Journal of Guidance, Control and Dynamics, Vol.10, pages 338-342, 1987.

[15] Bellman R. "Dynamic Programming".4th edition, Princeton University Press, 1965.

[16] S.G. Park, J.P. Clarke "Vertical Trajectory Optimization to Minimize Environmental Impact in the Presence of Wind". Georgia Institute of Technology, Atlanta, GA 30332. August, 2015.

[17] De Jong, P. M. A., De Gelder, N. and Bussink, F. J. L., Verhoeven, R. P. M., Kohrs, R. and Mulder, M. "Time and Energy Management during Descent and Approach for Aircraft: A Batch-Simulation Study". Journal of Aircraft. 2013
[18] E. Rippel ,A. Bar-Gill and N. Shimkin."Fast Graph-Search Algorithms for General Aviation Flight Trajectory Generation". Technion Israel Institute of Technology, Haifa, Israel. May, 2004.

[19] R. Devulapalli."An Efficient Algorithm for Commercial Aircraft Trajectory Optimization in the Air Traffic System”.University of Minnesota.July, 2012.

[20] R. Howe-Veenstra."Commercial Aircraft Trajectory Optimization and Efficiency of Air Traffic Control Procedures". University of Minnesota. November, 2011.

[21] A. Murrieta Mendoza, L.Ternisien, B.Beuze and R. Botez."Aircraft Vertical Route Optimization by Beam Search and Initial Search Space Reduction". Journal of Aerospace Information Systems. 2017-04-I010561. October, 2017.

[22] Hagelauer, P. and Mora-Camino, F. A. "Soft Dynamic Programming Approach for On-line Aircraft 4D Trajectory Optimization". European Journal of Operational Research, Vol. 10, 87-95, 1998.

[23] R. L.Schultz and N. R.Zagalsky. "Aircraft Performance Optimization". Honeywell Inc., Minneapolis. Journal of Aircraft, 1972.

[24] P.E. Hart, N.J. Nilsson and B. Raphael. "A Formal Basis for the Heuristic Determination of Minimum Cost Paths". IEEE Transactions of Systems Science and Cybernetics, Vol.SSC-4, No.2, July 1968. 